\title{
Les Goitres Plongeants : Aspects Épidémiologiques, Cliniques, Radiologiques Et Thérapeutiques
}

\author{
Illé $S$ \\ Service ORLet Chirurgie cervico-faciale \\ à l'hôpital national de Niamey, Niger \\ James Didié L \\ Service de chirurgie viscérale et digestive \\ à l'hôpital national de Niamey, Niger \\ Bako I \\ Service de Radiologie à l'hôpital national de Niamey, Niger \\ Sani Rachid \\ Service de chirurgie viscérale et digestive \\ à l’hôpital national de Niamey, Niger \\ doi: 10.19044/esj.2016.v12n30p305 URL:http://dx.doi.org/10.19044/esj.2016.v12n30p305
}

\section{Abstract}

Purpose: To describe the epidemiological and diagnostic aspects and surgical treatment procedures of plunging goiter in the Oto-RhinoLaryngology and Neck Surgery Office of the National Hospital of Niamey in Niger.

Materials and Methods: We analyzed the clinical and paraclinical symptoms as well as the technique and postoperative evolution of plunging goiters through a retrospective and descriptive study conducted from January 2010 to December 2015.

Results: During the five years period, 15 cases of plunging goiter were registered representing $6.35 \%$ of thyroidectomy. Among the patients, there were 4 men (26.66\%) and 11 women (73.34\%). The mean age was 37.84 years. The character "plunging goiter" was noticed on clinical examination and confirmed by imaging. Two cases of hyperthyroidism were recorded. Eso-trachea compression that is characterized by dysphagia and dyspnea was recorded in 7 cases (46.66\%), dysphonia in 2 cases (13.33\%). The scanner performed in 8 cases showed a goiter with anterior superior mediastinal shift in $84.61 \%$, driving the eso-tracheal axis in $62.5 \%$ of cases. The goiters plunged right in 5 cases (33.33\%), left in 2 cases (13.33\%) and were bilateral in 1 case. Complete thyroidectomy was performed on all patients by exclusive cervical way with as immediate complications, one intraoperative hemorrhage case $(n=1)$ and one transient hypoparathyroidism case $(n=1)$. 
Histological examination of surgical specimens shows no evidence of malignancy.

Conclusion: The plunging goiters are rare and their treatment was complete thyroidectomy by exclusive cervical way. Postoperative evolution was uneventful.

Keywords: plunging goiter, thyroidectomy, National Hospital of Niamey.

\section{Résumé}

But : Décrire les aspects épidémiologiques, diagnostiques et les modalités de prise en charge chirurgicale des goitres plongeants au service d'Oto-Rhino-Laryngologie et Chirurgie Cervico-Faciale de l'Hôpital National de Niamey au Niger.

Matériels et méthode: De janvier 2010 à décembre 2015, nous avons analysés les aspects cliniques, paracliniques et les suites post-opératoires des goitres plongeants grâce à une étude rétrospective et descriptive.

Résultats : En 5ans, 15 cas de goitre plongeant, représentant 6,35\% des thyrö̈dectomies étaient enregistrés chez 4 hommes (26,66\%) et 11 femmes (73,34\%). L’âge moyen était de 37,84 ans. Le caractère plongeant du goitre était noté à l'examen clinique ORL et confirmé par l'imagerie. Deux cas d’hyperthyroïdie étaient notés. Les signes de compression oesotrachéale caractérisés par la dysphagie et la dyspnée, existaient dans 7 cas (46,66\%), la dysphonie dans 2 cas (13,33\%).Le scanner cervico-thoracique réalisé dans 8 cas montrait un goitre à développement médiastinal antérosupérieur dans $84,61 \%$, refoulant l'axe oesotrachéal dans 62,5\% des cas. Le goitre plongeait à droite dans 5 cas (33,33\%), à gauche dans 2 cas $(13,33 \%)$ et était bilatéral dans 1 cas. La thyroïdectomie totale était réalisée chez tous les patients par voie cervicale exclusive avec comme seules complications immédiates, une hémorragie peropératoire $(n=1)$ et une hypoparathyroïdie transitoire $(n=1)$. L’examen histologique des pièces opératoires n’a relevé aucun signe de malignité.

Conclusion : Les goitres plongeants sont rares et leur traitement a été la thyroidectomie totale par voie cervicale exclusive. Les suites opératoires étaient simples.

Mots-Clés: Goitre plongeant, thyroïdectomie, Hôpital National de Niamey.

\section{INTRODUCTION}

Le goitre est une hypertrophie diffuse du corps thyroïde .Le caractère plongeant est déterminé par une limite inférieure qui atteint ou franchit le plan des vaisseaux sous-claviers (Sethom, 2005). Les goitres plongeants sont rares, leur fréquence varie de1 à 16, 6\% dans la littérature (Amor Ben, 
2014).Les goitres plongeants se singularisent par des aspects diagnostiques et chirurgicaux particuliers. Les risques majeurs sont la compression de l'œsophage, des vaisseaux sous-claviers, mais surtout de la trachée avec détresse respiratoire aigüe, mettant en jeu le pronostic vital du patient. Le but de ce travail est de décrire les aspects épidémiologiques, diagnostiques, et les modalités de prise en charge chirurgicale des goitres plongeants dans notre service.

\section{MATERIELS ET METHODE}

Il s’agissait d'une étude rétrospective, descriptive, réalisée dans le service d'ORL et Chirurgie Cervico-Faciale de l'hôpital national de Niamey (Niger), sur une période de 5 ans (2010 à 2015). L’étude a concerné tous les patients opérés d'un goitre plongeant en période d’euthyroïdie. Tous ces patients ont fait l'objet d'un examen clinique ORL complet et d'une nasofibroscopie. Les examens complémentaires prescrits comprenaient une radiographie cervico-thoracique de face, une échographie cervicale, un scanner cervico-thoracique, le dosage des hormones thyroïdiennes (T3, T4, TSH), la calcémie et un examen anatomopathologique de la pièce opératoire. Les paramètres étudiés sont d’ordre épidémiologique, clinique, paraclinique, thérapeutique et évolutif. Les paramètres épidémiologiques concernent l’âge et le sexe. Les paramètres cliniques et paracliniques concernent le motif et le délai moyen de consultation, les résultats des examens cliniques et des examens complémentaires. Les aspects thérapeutiques et évolutifs étaient en rapport avec la technique opératoire et les suites opératoires immédiates ( $\leq$ 7jours).

\section{RESULTATS}

\section{1-Aspects épidémiologiques:}

En 5 ans, 15 thyroïdectomies sur les 236 pratiquées ont concerné les goitres plongeants, soit une fréquence de 6,35\%. On notait 4 hommes et 11 femmes, soit un sex- ratio de 0,36. L'âge moyen était de 37,84 ans (extrêmes 29 et 61 ans).

\section{2-Aspects cliniques}

Le délai moyen de consultation était de 49 mois (extrêmes 18 mois et 30ans).Le motif de consultation était la tuméfaction thyroïdienne cervicothoracique antérieure chez tous les patients. Les signes de compression oesotrachéale à type de dysphagie et de dyspnée étaient retrouvés dans 7 cas (46,66\%). La dysphonie était rapportée dans 2 cas. Des signes d'hyperthyroïdie (amaigrissement, palpitation) étaient retrouvés chez 2 patients. La consistance du goitre était ferme, la peau recouvrante était saine dans les 15 cas (100\%) et le diamètre moyen était de $6 \mathrm{~cm}$ (extrêmes 5 et 15 
cm). L'examen clinique n’avait pas mis en évidence des adénopathies cervicales palpables chez tous les patients. La nasofibroscopie a mis en évidence une déviation du larynx vers la droite par compression chez 2 patients ; cependant, la mobilité des cordes vocales était conservée chez tous les patients.

\section{3-Aspects paracliniques}

La radiographie cervico-thoracique de face réalisée (figure1) a mis en évidence une opacité cervicale tissulaire descendant dans le thorax confirmant un goitre plongeant. Une déviation trachéale était notée chez $60 \%(n=9)$ des patients. L'échographie cervicale a montré un aspect hétérogène du goitre dans tous les cas. Seuls 8 patients sur les 15 ont pu réaliser le scanner cervico-thoracique. Cet examen mettait en évidence un goitre plongeant antérieur et postérieur respectivement dans 7 et 1 cas, refoulant l'axe oesotrachéal (figure2). Le goitre plongeait à droite dans 5 cas, à gauche dans 2 cas et bilatéral dans 1 cas (figure3). L'observation peropératoire retrouvait que le goitre était antérieur dans 14 cas (93,34\%) et postérieur dans 1 cas (6,66\%). Selon le coté, le goitre plongeait à droite dans 9 cas (60\%), à gauche dans 5 cas (33,34\%) et bilatéral dans 1 cas (6,66\%).Le dosage des hormones thyroïdiennes a révélé une hyperthyroïdie dans 2 cas. La calcémie préopératoire était normale chez tous les patients. L'examen histologique des pièces opératoires n’a relevé aucun signe de malignité.

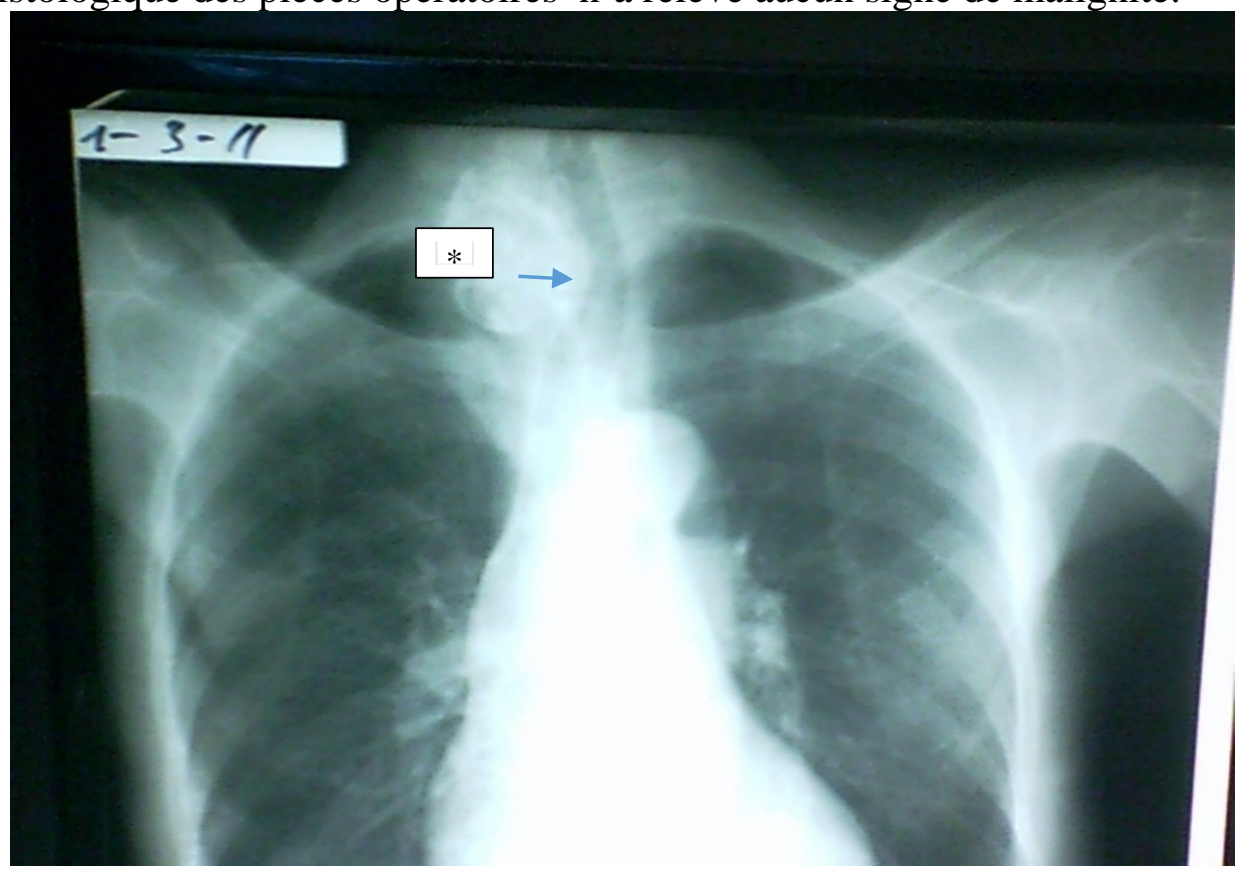

Figure1 : Radiographie cervico-thoracique montrant un goitre plongeant à droite $\left(^{*}\right)$ et déviant la trachéale à gauche ( $\rightarrow$ ). 


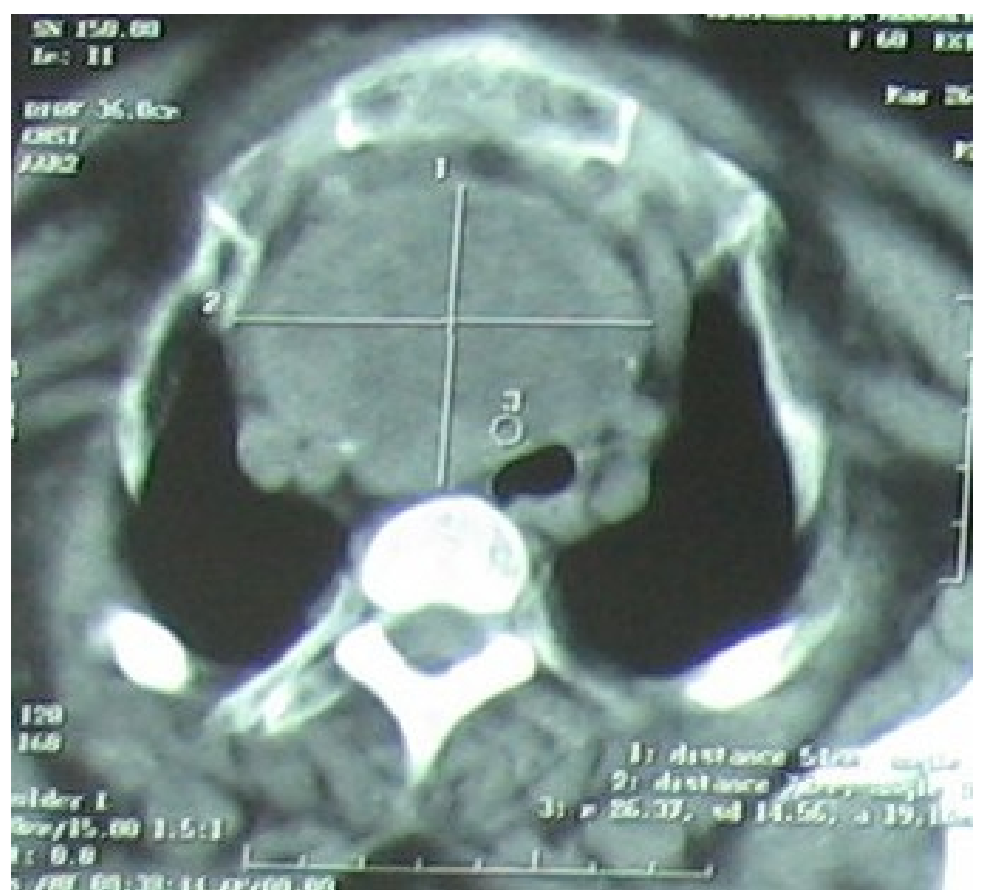

Figure2 : Scanner (coupe axiale) d'un goitre plongeant dans le médiastin supérieur et compression trachéale à gauche.

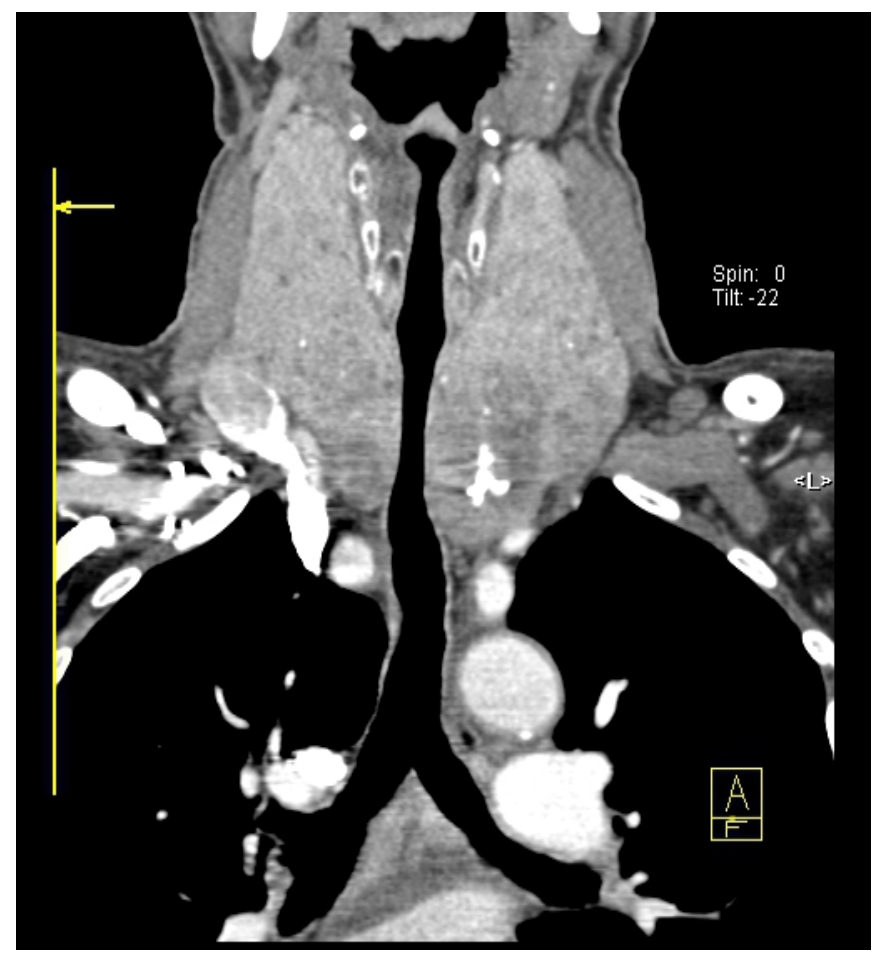

Figure3 : Scanner cervico-thoracique, coupe coronale montrant un goitre plongeant de manière bilatérale et un peu plus à gauche. 


\section{4-Aspects thérapeutiques}

La thyroïdectomie a été totale et exclusivement réalisée par voie cervicale. Le repérage visuel préalable du nerf récurrent a été systématique chez tous les patients. Les parathyroïdes ont été recherchées, vues et conservées dans tous les cas. Un cas d'hémorragie peropératoire a nécessité une transfusion sanguine iso groupe iso rhésus. A J1 postopératoire, un cas hypoparathyroïdie a été noté. Sa normalisation est intervenue à partir du quatrième jour sous perfusion de calcium. Les signes de compression oesotrachéale et laryngée ont régressé après l'exérèse du goitre. La mortalité post-opératoire immédiate était nulle. Tous les patients avaient bénéficié d'une hormonothérapie substitutive à vie.

\section{DISCUSSION :}

Les goitres plongeants sont rares et représentent 6,35\% de l'ensemble des thyroïdectomies réalisées en 5 ans dans notre expérience. La rareté des goitres plongeants est également rapportée dans certaines études (Amor Ben, 2014 Okiemy, 2004). L’âge moyen de 37,84 ans de notre série s’inscrit dans l'intervalle de 35 à 41 ans rapporté dans la littérature (Okiemy, 2004 Ouédé, 2010). Tout comme dans la littérature, une prédominance féminine a été constatée. Les goitres plongeants en particulier et la pathologie thyroïdienne en général sont en effet très fréquents chez la femme compte tenu des facteurs hormonaux (Makeieff, 2000 Taneri, 2005). Dans notre étude, le goitre plongeant était bénin dans tous les cas, tout sexe confondu. Ces données diffèrent de celles de la littérature où la constatation d'une tumeur thyrö̈dienne chez un homme doit faire craindre un cancer (Miccoli, 2006). L’incidence du cancer varie entre 2 et 19\% (Makeieff, 2000). Le délai moyen de consultation était long, excédant 4 ans. Il était de 52 mois dans la série d'Amor et coll (Amor Ben, 2014) en Tunisie.

L'imagerie concourt au diagnostic des goitres plongeants. La radiographie cervico-thoracique et l'échographie cervicale ont été de réalisation systématique dans notre pratique car peu onéreux et accessibles à tous nos patients. Ces deux examens ont confirmé le caractère plongeant du goitre déjà suspecté à l'examen clinique. L’évaluation précise du prolongement thoracique (topographie, rapports vasculaires) par le scanner cervico-thoracique a été possible chez 8 patients. La prédominance des cas de prolongement antérieur thoracique du goitre corrobore les données de la littérature en ce sens que les goitres plongeants postérieurs ne représenteraient que 10 à 15\% des cas (Zainine, 2011).

La descente du goitre dans le thorax est plus fréquente à droite qu’à gauche comme l'a également montré notre série. L’absence d’obstacle anatomique explique la progression du goitre vers le côté droit contrairement au côté gauche où la descente est gênée par les gros vaisseaux 
(Zainine, 2011). L’indication chirurgicale des goitres plongeants est formelle. Deux voies d'abord sont possibles en fonction de l'extension du goitre : soit la voie cervicale exclusive, soit la cervicotomie associée à la sternotomie ou la thoracotomie (Atoini, 2009). La sternotomie ou thoracotomie sont indiquées dans les goitres à prolongements multiples, croisés, à contingents solides, les reprises chirurgicales, les goitres postérieurs qui sont surtout susceptibles d'entrainer des difficultés opératoires, ou après échec d'une tentative d'extraction par voie cervicale (Grainger, 2005). Nous avons réussi l'exérèse totale de la glande par voie cervicale exclusive après repérage visuel et dissection du nerf récurrent. Le prolongement médiastinal antéro-supérieur majoritaire et l'accouchement au doigt sans difficulté ont favorisé la restriction à la voie cervicale utilisée. Les suites postopératoires sont globalement satisfaisantes car seul un cas d'hémorragie peropératoire et un cas d'hypoparathyroidie transitoire a été enregistré. La morbidité de la thyroïdectomie pour goitre plongeant est identique à celle de toute chirurgie thyroïdienne (Dieng, 2010 Batori, 2005). Le risque d'hypoparathyroidie est par contre plus important que dans la chirurgie des goitres cervicaux (Amor Ben, 2014). En effet, le repérage de la glande parathyroïde peut s'avérer difficile, en particulier pour la parathyroïde inférieure souvent accolée à la face profonde du goitre et en position très basse à la jonction cervico-thoracique (Amor Ben, 2014).

\section{Conclusion}

Dans notre pratique, Le goitre plongeant est une pathologie thyroïdienne rare, touchant plus la femme que l'homme. Le diagnostic est clinique et est confirmé par l'imagerie grâce à une simple radiographie thoracique. La thyroïdectomie totale par voie cervicale exclusive a été la principale modalité chirurgicale des goitres plongeants à Niamey. Les suites opératoires immédiates, en majorité, simples.

\section{References :}

1. Sethom A, Brahem H, Ouni $\mathrm{H}$ et coll. (2005). Les goitres plongeants. A propos de 15 cas. JTUN ORL, 14, 21-24.

2. Amor Ben S, Dhambri S,Hariga I et coll. (2014). Les goitres plongeants: Particularités Cliniques, radiologiques et thérapeutiques. JTUN ORL, 31,27-29.

3. Okiemy G, Elé N, N'Gouoni GB, Gombet T, Kipamboudi A. (2004). Les goitres plongeants. A propos de 27 cas. Médecine d'Afrique Noire, 51(3) ,167-175.

4. Ouédé R, Demine B, Kendja F, Kouamé J, Paul Yapo Y, N’Guessan Joël $G$ et al. (2010). Goitre plongeant endothoracique. Le Journal Africain du Thorax et des Vaisseaux, 00(00), 22-26. 
5. Makeieff M, Marlier F, Khudjadze M, Garrel R, Crampette L, Guerrier B.(2000). Substernal goiters: Report of 212 cases. Ann chir, 125(1), 18-25.

6. Taneri F, Kurulahvecioglu O, Ege B, Yilmaz U, Tekin E, Cifter C et al .(2005).Prospective analysis of 518 cases with thyroidectomie in Turkey.EndocrRegul,39(3),85-90.

7. Miccoli P, Berti P, Frustaci GL, Ambrosini CE, Materazzi G. (2006). Video-assisted thyroidectomy: indications and results. Langenbecks Arch Surg, 391(2), 68-71.

8. Zainine R,ElAoud C, Bacharaoui R, Beltaief N, Sahtout S, Besbes G.(2011). The plunging goiter: about 43 cases. La Tunisie Médicale, 89(11), 860-865.

9. Atoini F, Zidane A, Traibi A, Arsalane A, Elkaoui H, Tahri N et al. (2009). Surgical management of substernal goiters : a series of 27 patients. J Chir, 146(2), 229-31.

10. Grainger $\mathrm{J}^{1}$, Saravanappa N, D'Souza A, Wilcok D, Wilson PS.(2005). The surgical approach to retrosternal goiters: the role of computerized tomography. Otolaryngol Head Neck Surg, 132, 84951.

11. Dieng M, Cissé M, Ndour MD, Konaté I, Touré AO, Ka O et coll. (2010). Indications et résultats des thyroïdectomies réalisées au sein d'un service de chirurgie générale. A propos de 402 patients opérés. Revue Africaine de Chirurgie et Spécialités, 4(9) ,24-27.

12. Batori M, Chatelou E, Straniero A et al.(2005). Substernal goiters.Eur Rev Med Pharmacol Sci, 9,355-9. 